\title{
Observation of long-range correlations in temporal intensity fluctuations of light
}

\author{
Frank Scheffold \\ Institut Charles Sadron, CNRS, 6 rue Boussingault, F-67083 Strasbourg Cedex, France \\ Wolfram Härtl \\ Physikalische Chemie, Universität des Saarlandes, D-66123 Saarbrücken, Germany \\ Georg Maret \\ Fakultät für Physik, Universität Konstanz, Postfach 5560, D-78457 Konstanz, Germany
}

Egon Matijević

Center for Advanced Materials Processing, Clarkson University, Box 5814, Potsdam, New York 13699-5814

(Received 13 May 1997)

\begin{abstract}
We present evidence for long-range correlations $C_{2}(t)$ in temporal intensity fluctuations of multiply scattered visible light. The time autocorrelation function of the angular-averaged light intensity transmitted through a thin slab containing particles undergoing Brownian motion was determined for a series of different sample thicknesses, beam spot sizes at the sample surface, and optical transport mean free paths $l^{*}$. The results for both the amplitude (i.e., the inverse conductance $1 / g$ ) as well as the time $(t)$ dependence of the correlation function $C_{2}(t)$ are in good overall agreement with theory. Significant deviations are found, however, when correlations generated in a layer of $1 l^{*}$ to $2 l^{*}$ adjacent to the incoming surface become important. In particular the predicted $t^{-1 / 2}$ long-time tail of $C_{2}(t)$ was not observed. A physical explanation of these deviations is given based on the argument that at least one scattering event is required before long-range correlations $C_{2}(t)$ can develop. [S0163-1829(97)08341-0]
\end{abstract}

\section{INTRODUCTION}

Within the last decade light propagation in random media has attracted considerable attention. In analogy to electronic transport in disordered metals, fundamental issues such as localization of classical waves have been addressed. ${ }^{1}$ After the discovery of weak localization of light, ${ }^{2,3}$ a precursor of light localization, several effects have been studied (see, e.g., Refs. 4-7). It was shown both theoretically and experimentally that, despite the randomness of the medium, various interference effects are essential for the light propagation in the multiple-scattering regime.

Long-range correlations in the fluctuations of the scattered intensity ("'speckle") represent one of the most interesting examples. Due to particular interference effects caused by the crossing of scattering paths inside the random medium, two types of correlations between different speckle spots build up..$^{8-10}$ (1) Long-range correlations in the scattered fields give rise to fluctuations in the angular-integrated transmission (for plane-wave illumination of the sample). In the following we will refer to this case as "integrated transmission." (2) Infinitive range correlations cause fluctuations in the transmission even for diffuse illumination. The latter fluctuations are considered the optical analog of the "universal conductance fluctuations (UCF)', in electronic systems. The only observation so far of optical long-range correlations was made by measurements of the light intensity transmitted through a solid turbid medium as a function of a frequency shift. ${ }^{11,12}$ Long-range correlations have also been observed in multiple scattering of microwaves. ${ }^{13}$

In this paper we present the observation of long-range correlations in temporal intensity fluctuations. A Gaussian laser beam is strongly focused on a thin slab containing a suspension of colloidal particles undergoing Brownian motion. We have measured the time $(t)$ autocorrelation function $C(t)=\langle I(0) I(t)\rangle /\langle I(0)\rangle^{2}-1$ of the angular-averaged light intensity transmitted through the slab. After a brief review of existing theory, we present a detailed experimental analysis of the temporal shape of the correlation function and its amplitude dependence on sample thickness, beam spot size, and transport mean free path $l^{*}$. Because of the inherently small noise level in dynamic light-scattering experiments, these measurements provide access to a much wider range of parameters than achieved before, as will be discussed in detail in the experimental section.

\section{LONG-RANGE SPECKLE CORRELATIONS}

\section{A. The physical picture}

Light propagation in optically thick, i.e., "turbid,' random media is usually described by diffusive transport. ${ }^{4-7}$ Incident light is fully randomized after a typical distance of one transport mean free path $l^{*}$ and propagates diffusively inside the medium. The diffusion process is completely characterized by $l^{*}$. The physically most intuitive picture to describe the transport of the scattered light intensity is the random walk of photons. All photons are scattered along statistically independent random scattering paths $s$. This allows us to calculate easily the path length distribution $P(s)$ of the diffusing photons for a given geometry. This picture does not, however, account for the interference effects which are the origin of the speckle pattern. The latter arise from 
interferences between the electric fields scattered along different scattering paths as indicated in Fig. 1(a), showing a snapshot of two scattered fields at a given instant of time (taken as 0 ), and a small time $t$ later. The relative phase between $E_{i}$ and $E_{j}^{*}$ fluctuates randomly in time because of the random motion of particles and the randomness of the scattering paths. This gives rise to the temporal fluctuations of the total intensity $I=\left\langle\Sigma_{\text {paths }} E_{i} E_{j}^{*}\right\rangle^{2}$ scattered in a given direction, that is into a far-field speckle spot. It is important to note, however, that for totally uncorrelated random scattering paths, fields $E_{i}$ and $E_{j}$ are uncorrelated, and hence all terms $\left\langle E_{i} E_{j}^{*}\right\rangle$ vanish, when \langle\rangle denotes the average over time or over many configurations of the positions of the scattering particles. This has two consequences: First, the average total scattered intensity $\langle I\rangle$ is just the sum of the average intensity scattered along the individual paths. Second, the intensity correlator $\langle I(0) I(t)\rangle$ becomes $\left\langle\Sigma_{\text {paths }} E_{i}(0) E_{i}^{*}(t)\right\rangle^{2}$ containing only contributions of field autocorrelations along the individual paths, but no cross terms between different paths. This tremendously simplifies the description of dynamic multiple light scattering and is the key for the recent success of diffusing wave spectroscopy (DWS) ${ }^{16}$ One of the important features of DWS, which becomes physically very transparent in the above picture, is that long/short scattering paths provide fast/slow fluctuations of $I$, respectively. This follows from the fact that the time-dependent phase shifts between $E_{i}(0)$ and $E_{i}(t)$ increase with the number of scattering events in a path and hence with its length.

Feng and co-workers ${ }^{8,9}$ showed that the assumption of independent paths omits interesting contributions to $C(t)$. There is a finite probability that two light paths cross each other at a certain site, as indicated schematically in Figs. 1(b) and $1(\mathrm{c})$. After the crossing there are two nonvanishing contributions of equal weight to $C(t)$. The first case [Fig. 1(b)] correlates $E_{i}(0)$ with $E_{i}(t)$ and $E_{j}(0)$ with $E_{j}(t)$ and therefore has the same features as for independent paths [Fig. 1(a)]. In particular the intensity fluctuations of different speckle spots are uncorrelated. If, however, as indicated in Fig. 1(c), we exchange $E_{i}(t)$ with $E_{j}(0)$, the fields scattered along the paths after the crossing point remain pairwise correlated for all correlation times $t$ and hence do not contribute to the decay of $C(t)$. In addition, since the interference arises from fields scattered along the same scattering path, this correlation is independent of the angle of observation. This second contribution therefore leads to small fluctuations of the angular-integrated transmission. Decorrelations occur only before the crossing point [left-hand side of Fig. 1(c)] and therefore the "active" sections of the paths are shorter and the distribution of path lengths is much broader as compared to the case of independent scattering paths [Fig. 1(a)]. The magnitude of the effect $(1 / g)$ is given by the crossing probability of two paths at some point inside the sample.

In the less probable case of two crossing events along a pair of paths, additional correlations arise ${ }^{8}$ which lead to fluctuations independent of the direction of the incident and the outgoing fields. These very slow and small fluctuations of the total transmission (or optical conductance) $C_{3}(t)$ are considered the optical analog of the universal conductance fluctuations (UCF) in electronic systems. a)

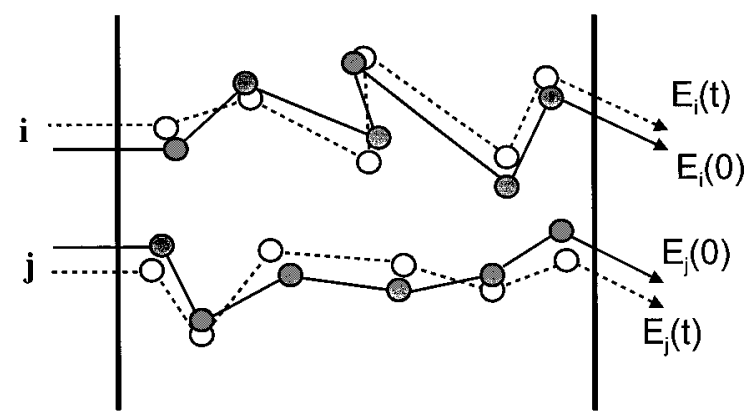

b)

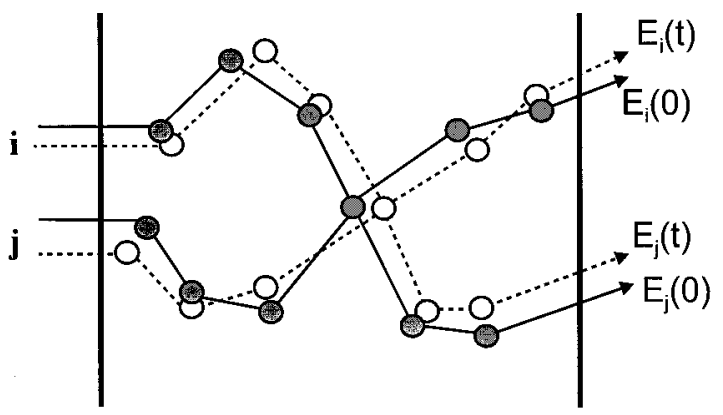

c)

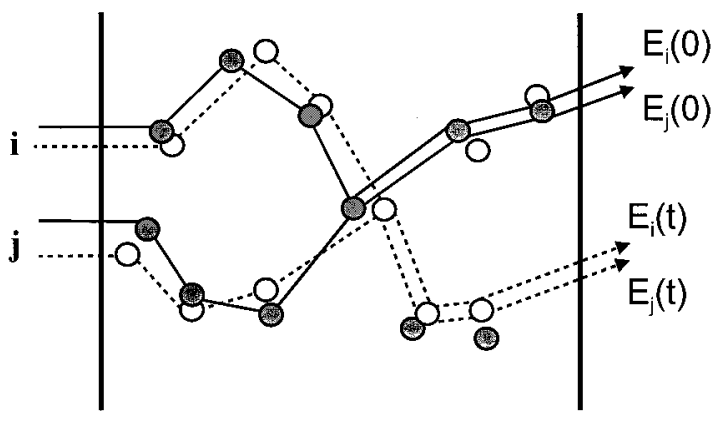

FIG. 1. Physical origin of long-range intensity correlations: In a turbid sample light propagation can be described as a random walk of photons. In order to describe interference effects one has to consider the electric fields scattered by the particles (full circles) along different paths $i, j$. Most of the light paths in a disordered medium are independent (a) and give therefore rise to a pattern of uncorrelated so-called "speckle" spots. Due to the Brownian motion of the particles (open circles) in a dense colloidal suspension, phase shifts are introduced between the electric fields at a given time $t=0$ and at a certain time $t$ later which leads to characteristic fluctuations $C_{1}(t)$ of individual speckle spots. Crossing of light paths inside the sample (b),(c) provides a type of correlation which does not average to zero in the intensity correlator $\langle I(0) I(t)\rangle$. While in the first case (b) we recover the same characteristics for the scattered light as for independent paths (a), a totally different effect appears in the case where after the crossing point the electric fields $E_{i}, E_{j}$ at a given time are propagating along the same path (c). In this particular case the light fields $E_{i}, E_{j}$ are completely correlated along each remaining scattering path and no further dephasing occurs for all times $t$. This results in a slowly fluctuating angular-independent contribution $C_{2}(t)$ to the transmitted light intensity.

\section{B. The conductance $g$}

Using a diagrammatic technique Feng et al. ${ }^{8}$ considered a cylindrical waveguide of diameter $W$ with perfectly reflecting walls. The average intensity, which is transmitted from an incoming plane light wave (mode $a$ ) to an outgoing planewave mode $b$, is called $\left\langle T_{a b}\right\rangle$. The (dimensionless) conduc- 
tance $g$ of the sample is then defined as the sum over all incoming and outgoing modes:

$$
g \equiv \sum_{a, b}\left\langle T_{a b}\right\rangle
$$

It has been shown ${ }^{8}$ that $1 / g$ also describes the probability that two paths cross somewhere inside the sample. Hence the intensity autocorrelation function can be written in terms of contributions of zero crossings $C_{1}(x)$, one crossing $C_{2}(x)$, and two crossings $C_{3}(x), x$ being some quantity, such as frequency shift $\Delta \omega$ or correlation time $t$, which introduces phase shifts between optical fields:

$$
\begin{gathered}
C(x)=C_{1}(x)+\frac{1}{g} C_{2}(x)+\frac{1}{g^{2}} C_{3}(x), \\
g=\frac{N l^{*}}{L} \sim W^{2} .
\end{gathered}
$$

$N$ is the number of modes inside the waveguide of thickness $L . N$ is proportional to $W^{2}$ and thus $g$ is proportional to the surface area of the sample. Here the conductance $g$ is independent of the functional behavior of $C_{2}(x)$ and $C_{3}(x)$, i.e., it is independent of the nature of $x$.

Because of the difficulties in realizing experimentally a small optical waveguide with perfectly reflecting walls, we choose for our experiments a slightly different setup, as already suggested by Feng et al. ${ }^{8}$ A thin slab of a colloidal suspension $(L \approx 10-100 \mu \mathrm{m})$ was illuminated with a strongly focused Gaussian beam with transverse intensity profile:

$$
I(r)=\frac{2}{w^{2} \sqrt{\pi}} \exp \left(-\frac{4 r^{2}}{w^{2}}\right)
$$

The beam waist $w(w \approx 3-60 \mu \mathrm{m})$ is defined by the distance between the $1 / e$ points of the transverse intensity distribution. In this case the theory of Feng et al. ${ }^{8}$ should hold in the limit of $w \gg L$.

Pnini and Shapiro ${ }^{14}$ extended the theory to the general case of a finite beam spot incident on a slab. They calculated the conductance $g$ for a homogeneous beam spot of size $W$, with $I(r)=1$ for $0 \leqslant r<W / 2$ and $I(r)=0$ otherwise. For the case $W \ll L$, they find that the conductance $g$ scales linearly with the beam spot size $g \sim W$. Later de Boer et al. ${ }^{12}$ generalized this result for an incident Gaussian beam, usually encountered in optical experiments. Although they considered frequency correlations $C(\Delta \omega)$ their results for $g$ apply to the case of temporal correlations as well because of the insensitivity of $g$ on the phase shift introducing parameter:

$$
\begin{gathered}
\frac{1}{g}=\frac{1}{\alpha} \times \frac{L}{w^{2}} \times F\left(\frac{w}{L}\right), \\
\alpha=\frac{k_{0}^{2} l^{*}}{3}=\frac{l^{*}}{3}\left(\frac{2 \pi n}{\lambda}\right)^{2},
\end{gathered}
$$

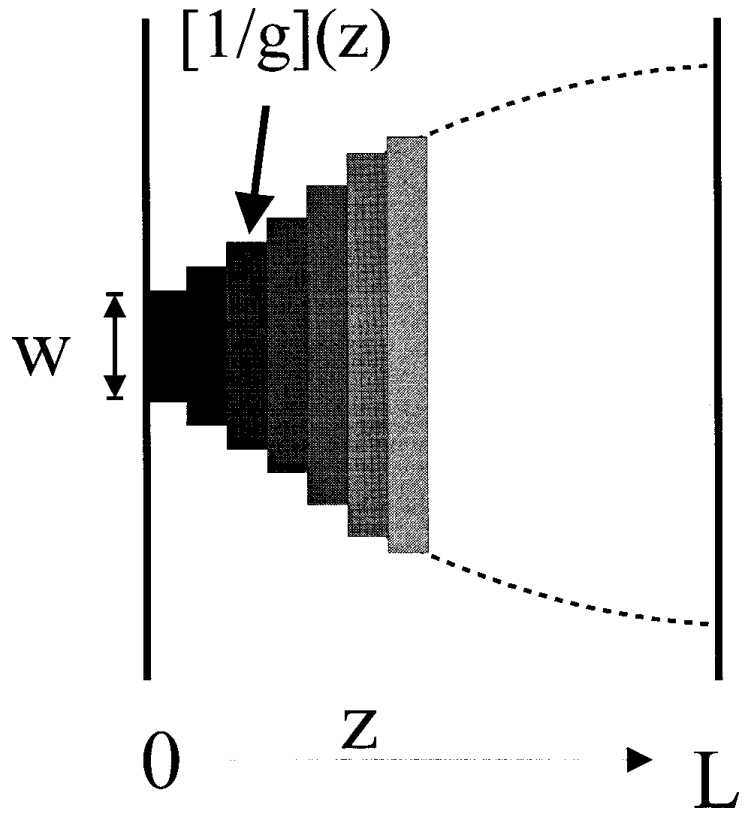

FIG. 2. Broadening of the photon cloud inside the sample of thickness $L$ leads to a sharp decline of the "crossing probability" $[1 / g](z)$ with increasing depth $z$. If the beam spot size is $w \ll L$, crossing events close to the incoming surface have a much higher weight and dominate the conductance $g$ and the temporal shape of the correlation function since the effective path lengths before a crossing event are much shorter as compared to a cylindrical waveguide of identical thickness $L$. With increasing beam spot size $w$ the decay of the crossing probability becomes less sharp until in the case $w \gg L$ it is independent of the depth $z$.

$$
F\left(\frac{w}{L}\right)=\int_{0}^{\infty} d x\left(\frac{w}{L}\right)^{2} \exp \left[-\left(\frac{w}{L}\right)^{2} \frac{x^{2}}{32}\right] \frac{x[\sinh (x) / x-1]}{8[\cosh (x)-1]},
$$

where $n$ is the refractive index, and $\lambda$ is the wavelength of the incident light. In the limit $w \gg L, F(\infty) \rightarrow 2 / 3$, hence $g \sim w^{2}$.

There is a simple physical explanation for the linear dependence of $g / L$ on $w / L$ in the limit of $w / L \ll 1$. Due to the diffuse spreading of the multiply scattered light inside the sample, the crossing of two paths becomes less and less likely with increasing distance from the surface of incidence (Fig. 2). If we consider the conically shaped cloud of diffusing photons as a succession of thin slabs, an effective crossing probability (which is the inverse conductance) can be defined:

$$
1 / g(z) \sim 1 / w(z)^{2} \approx 1 /(w+\beta z)^{2}
$$

with $\beta$ of order $1 .{ }^{15}$ Integration over $z$ yields

$$
\begin{gathered}
\frac{1}{g} \sim \int_{0}^{L} \frac{1}{(w+\beta z)^{2}} d z=\frac{1}{w^{2} / L+\beta w} ; \quad w \ll L, \\
\frac{g}{L} \sim \frac{w}{L} .
\end{gathered}
$$




\section{Time dependence of the intensity correlation function $C(t)$} for Brownian scatterers

First we discuss briefly the well-known case of the light transmission through a slab $(w \gg L)$ containing Brownian particles. For this case the leading contribution to the intensity-intensity correlation function $1+C_{1}(t)$ can be derived from diffusion theory. ${ }^{16}$ Assuming light propagation on independent scattering paths [Fig. 1(a)], it is possible to calculate the actual distribution of light paths $P(s)$ and therefore $C_{1}(t)$. In the case of an incident plane wave $C_{1}(t)$ is then given by

$$
C_{1}(t)=\left(\frac{\left(L / l^{*}+4 / 3\right) \sqrt{6 t / \tau_{0}}}{\left(1+8 t / 3 \tau_{0}\right) \sinh \left[L / l^{*} \sqrt{6 t / \tau_{0}}\right]+4 / 3 \sqrt{6 t / \tau_{0}} \cosh \left[L / l^{*} \sqrt{6 t / \tau_{0}}\right]}\right)^{2}
$$

It was shown experimentally ${ }^{17}$ that this relation holds very well for samples of thickness $L$ larger than 8-10 transport mean free paths $l^{*}$. Here $\tau_{0}=1 / D k_{0}^{2}$ denotes the single scattering decay time and $D$ is the translational diffusion constant of the scatterers.

The correlation function is dominated by a typical path length $L^{2} / l^{*}$ of the diffusing light where each scattering event contributes on average by $\exp \left(-t / \tau_{0}\right)$ to the decay of the correlation function. Hence $C_{1}(t)$ decays essentially exponentially with

$$
C_{1}(t) \cong \exp \left(-2\left(\frac{L}{l^{*}}\right)^{2} \frac{t}{\tau_{0}}\right)
$$

The derivation of the correlation function $C_{2}(t)$ is less straightforward. $C_{2}(\Delta \omega)$ was calculated by de Boer et al. ${ }^{12}$ but unlike the conductance $g$, the dynamical part of the longrange correlations $C_{2}(\Delta \omega)$ cannot be directly transferred to the time domain $C_{2}(t)$. Instead one would have to apply the theory ${ }^{12}$ to the case of Brownian scatterers, which is beyond the scope of this work.

To our knowledge the only theoretical treatment of longrange $C_{2}(t)$ correlations for Brownian scatterers is presented in a paper by Berkovits and Feng. ${ }^{10}$ Using a diagrammatic technique they derive the intensity-intensity correlation function for the case $w \gg L$ and find

$$
\begin{gathered}
C_{2}(t)=\frac{3}{2 \sqrt{6 \frac{t}{t_{0}}}}\left[\operatorname{coth}\left(\sqrt{6 \frac{t}{t_{0}}}\right)-\frac{\sqrt{6\left(t / t_{0}\right)}}{\sinh ^{2}\left(\sqrt{6\left(t / t_{0}\right)}\right)}\right], \\
t_{0} \approx\left(\frac{l^{*}}{L}\right)^{2} \tau_{0} .
\end{gathered}
$$

The $C_{2}(t)$ correlation function decays over a much broader time scale than in the case of short-range $C_{1}(t)$ correlations. In the long-time limit an algebraic $t^{-1 / 2}$ behavior is predicted.

A problem always encountered in the description of light propagation in random media is the coupling of light from the outside to the inside of the sample. Outside the sample the light can be described by superposition of plane waves, while inside the sample the light propagates in the diffusive regime. Virtually all theories introduce an injection source at a depth $z \approx l^{*}$ and an ejection drain at $z \approx L-l^{*} \cdot{ }^{4-7}$ In this crude approximation all diffuse intensity is generated at injection depth $z \approx l^{*}$ with respect to the surface of the slab. This approximation yields excellent agreement with experiment in the case of $C_{1}(t)$ correlations in transmission, ${ }^{16}$ where the contribution of short scattering paths is small. For measurements in backscattering geometry the agreement is still good, with a parameter $\gamma$ of order 1 , which depends somewhat on the system characteristics and scattering geometry. $^{16}$

We emphasize here that for long correlation times, i.e., short scattering paths, the $C_{2}(t)$ correlations are very sensitive to the region near the surface. As illustrated in Fig. 3 the effective depth at which scattering, i.e., dephasing of the light, starts to take place does not coincide with the effective depth at which the first crossing event [generating $C_{2}(t)$ correlations] may first occur. The light plane-wave incident on the slab has to be scattered at least once before two distinct light paths can cross. The correlated light emerging from scatterer 2 shows dynamic fluctuations caused by the motion of scatterers 1 and $1^{\prime}$, which yields a cutoff at long correlation times in $C_{2}(t)$ and is not taken into account in present theories. Based on this argument we expect downward deviations from the predicted $t^{-1 / 2}$ law.

Furthermore, we recall that the conductance $g$ depends

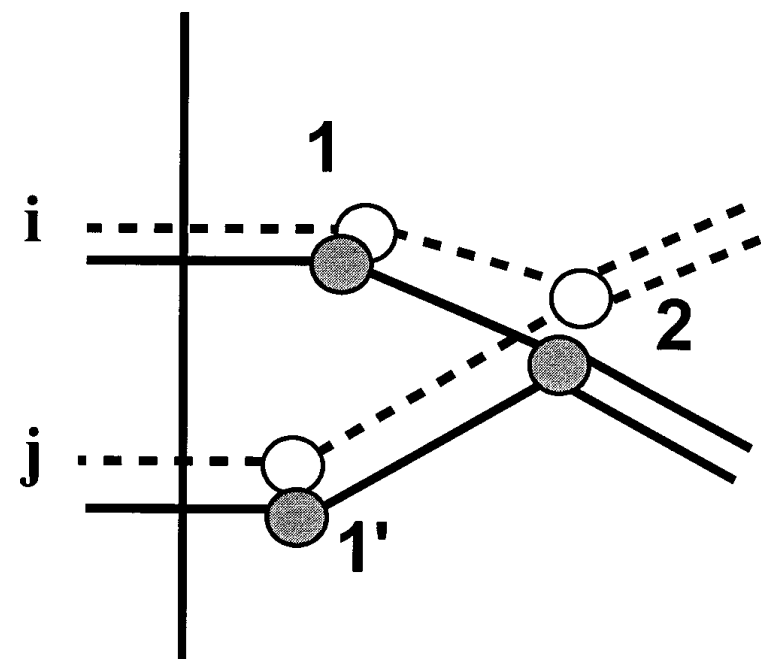

FIG. 3. Light incident on the sample is scattered at least once before it contributes to the long-range $C_{2}(t)$ correlations. 
strongly on the lateral confinement of the scattering volume, i.e., the beam spot size $w$ [Eq. (5)]. Therefore a change in the size of the beam spot due to scattering in a layer near the surface before the light contributes to the $C_{2}(t)$ correlations has an influence on the magnitude of $g$, which is small as long as $w \gg l^{*}$. This suggests that the study of long-range correlations in the transmission of light through thin films probes very sensitively the vicinity of the surface.

\section{EXPERIMENT}

\section{A. Experimental setup and data analysis}

In order to be able to detect $C_{2}(t), C_{1}(t)$ has to be averaged by angular averaging over the outgoing speckle pattern. In addition, as seen from Eq. (5), it is necessary to realize a small sample size and $l^{*}$. In order to prepare thin slabs of a colloidal suspension we manufactured glass cells of different thicknesses using a simple etching technique. The center area (diameter $5 \mathrm{~mm}$ ) of a microscope cover plate (diameter 14 $\mathrm{mm}$ ) was exposed to a $0.6-2 \% \mathrm{HF}$-acid solution in order to create a uniform cylindrical hole. By varying the exposure time and solution strength, we could roughly control the cell volume. Filling the glass cells with a colloidal suspension and mounting a second glass plate onto it allowed us to prepare thin slabs of thickness 5 to $50 \mu \mathrm{m}$. Slabs thicker than $50 \mu \mathrm{m}$ where obtained using spacers. The typical roughness of the etched surface, as determined by atomic-force microscopy, was approximately $5-10 \mathrm{~nm}$, which is negligible as compared to the sample thickness, or even the minimum mean free path of the suspension $\left(l^{*}=0.98 \mu \mathrm{m}\right)$.

To prevent the suspension from drying, the glass plates were pressed against each other in the sample holder (Fig. 4) and sometimes additionally sealed with silicon. The fluctuations of the integrated transmission were measured with the setup illustrated in Fig. 4. A laser beam (diameter roughly 1 $\mathrm{mm}$ ) from an Ar-laser operating in single frequency mode at $457.9 \mathrm{~nm}$ was focused onto the sample. To obtain small beam spot sizes, we used either an optical lens of a focal length of $5 \mathrm{~cm}$, which yields a minimum beam spot size of $w=11.6 \mu \mathrm{m}$, or in one case a microscope objective to obtain beam spots down to $w=3.4 \mu \mathrm{m}$. This setup allowed us to change the actual beam spot size by variation of the sample-lens distance. The size of the beam spot $w$ was determined by replacing the sample by a $10 \mu \mathrm{m}$ pinhole, or a 1 $\mu \mathrm{m}$ pinhole in the latter case, and scanning across the beam (accuracy $\sim 5 \%$ ).

The glass cell was mounted in a sample holder and placed into an integrating sphere in order to average scattering intensities over all scattering angles of the transmitted light. A thick fiber bundle (diameter $5 \mathrm{~mm}$ ), positioned perpendicular to the incoming beam, was used to conduct the transmitted light from the integrating sphere to a photomultiplier. The fluctuations of the integrated transmission were analyzed using a commercial photon correlation setup. The multiple $\tau$ correlation scheme implemented in this correlator provides a fixed distribution of correlation times, which essentially consists of blocks of eight linearly spaced channels, with exponentially increasing correlation times from block to block. For illustration purposes we reduced the number of points of each block from eight to two by averaging the contents of

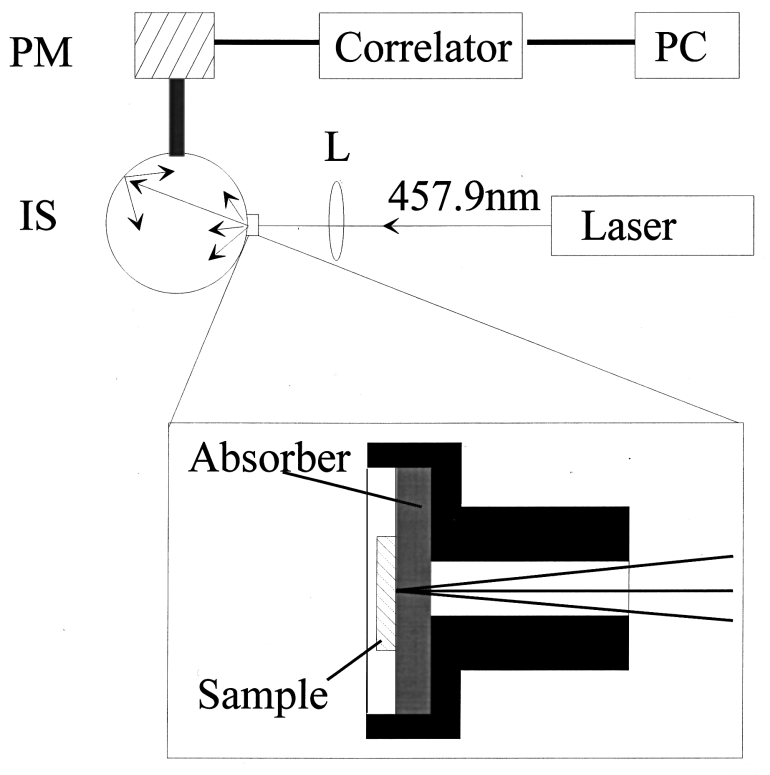

FIG. 4. The fluctuations of the integrated transmission through a slab were measured by angular averaging the transmitted light with an integrating sphere (IS). Detected by a photomultiplier unit (PM) the correlation function was subsequently analyzed using a digital correlator. An enlarged view of the sample holder shows the sample cell mounted onto an absorber, thus eliminating light reflected back from the incoming surface. The incident laser beam is strongly focused by a lens (L).

channels 1-4 and 5-8 of each block. Since all $C_{2}(t)$ functions decay very slowly the error introduced by this procedure is negligible.

The intrinsically low $1 / g$ values encountered in this experiment necessitated the measurements to be carried out at high photon count rates in order to improve the count rate statistics, which however caused systematically lower values of $1 / g$ due to dead-time effects. At the chosen count rate of $10( \pm 1.5) \mathrm{MHz}$, the difference is found to be $(9 \pm 4) \%$ based on measurements at count rates between 2.5 and 25 MHz. In order to correct for this we divided all $C_{2}(t)$ correlation functions by a factor of 0.91 . The deviation from a linear response of the photomultiplier setup was determined to be of the same order, i.e., $10-15 \%$ at this count rate. No detectable influence on the temporal shape of the $C_{2}(t)$ correlations was found. These observations are in qualitative agreement with a theoretical treatment of dead-time effects on photon-correlation functions by Schätzel et al. ${ }^{18}$

To determine the background noise signal, i.e., no longrange $C_{2}$ contribution, we measured the intensity-intensity correlation function without sample. For sufficiently large $w$ values no difference was detected to an otherwise identical experiment with the sample, indicating that the latter does not introduce further noise. The very small structure in this correlation function (Fig. 5) was reproducible in time and depended somewhat on the laser power. In general the highest value of the normalized noise-correlation function was in all cases less than $\left|\langle I(0) I(t)\rangle /\langle I(0)\rangle^{2}-1\right|<5 \times 10^{-6}$ for small correlation times $4 \times 10^{-7} \mathrm{~s}<\tau<10^{-5} \mathrm{~s}$ and further decaying down to an undetectable level for larger correlation times. Compared to the measured $C_{2}(t)$ functions the noise level is typically less than $5 \%$ over the whole range of cor- 


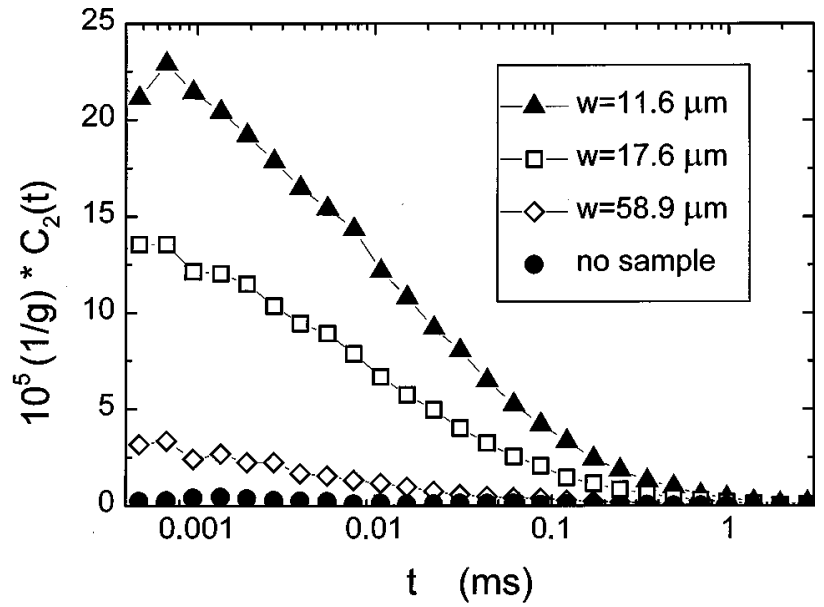

FIG. 5. Time autocorrelation function of the integrated transmission for different beam spot sizes $w$. The sample thickness is $L=36.7 \mu \mathrm{m}$ and the transport mean free path $l^{*}=0.98 \mu \mathrm{m}$.

relation times, rising to approximately $10 \%$ only for very large $w$ values, i.e., small $1 / g$, where the scatter of $C_{2}(t)$ is of the same order. Nevertheless we corrected the $C_{2}(t)$ functions for this error in order to improve accuracy, which was carried out by substraction of a measured noise-correlation function. In some cases we observed a nonzero baseline caused by slow fluctuations due to temperature variations or intrinsic remaining fluctuations of the laser intensity. This problem was suppressed by running multiple short runs, e.g., $300 \times 5 \mathrm{~s}$, to very low levels of $\mid\langle I(0) I(t \approx 50 \mathrm{~ms})\rangle /$ $\langle I(0)\rangle^{2}-1 \mid<2 \times 10^{-7}$. The duration of the runs was chosen to be still much larger than the decay time of $C_{2}(t)$.

\section{B. Sample characterization}

The magnitude of the $C_{2}(x)$ correlations depends strongly on the transport mean free path $l^{*}$ [Eq. (5)]. To maximize the signal, it is desirable to prepare a suspension of minimum $l^{*}$. Among weakly or nonabsorbing particles colloidal $\mathrm{TiO}_{2}$ has the highest refractive index $n \approx 2.7$; however, it is difficult to synthesize sufficient amounts of calibrated monodisperse samples. We therefore decided to use colloidal $\mathrm{BaTiO}_{3}$ with a refractive index $n=2.4$ and a rather small polydispersity of $\Delta d / d \approx 20-30 \% .{ }^{19}$ The average particle diameter of $d=(208 \pm 6) \mathrm{nm}$ was determined by standard photon correlation spectroscopy at a scattering angle of $\Theta=90^{\circ}$. To prepare stable aqueous suspensions we had to adsorb polyacrylic acid (PAA, $M_{w}=2000$ ) to a saturation level onto the colloid surface. ${ }^{20}$ At $\mathrm{pH} \approx 9$ this additional polymer layer introduces an effective barrier against particle aggregation. In doing so, we were able to prepare stable samples with volume fractions up to $38 \%$.

For spherical particles of sufficiently small concentration, i.e., noninteracting particles, $1 / l^{*}$ is found to scale proportional to the volume fraction $\Phi$ of the particles. In this regime it is possible to determine $l^{*}$ accurately by measuring the intensity-intensity correlation function $C_{1}(t)$ in transmission, since the single scattering decay time $\tau_{0}=1 /\left(D k_{0}^{2}\right)=1.4 \mathrm{~ms}$ can be used [see Eq. (11)]. However, for higher volume fractions the scatterers cannot be treated as noninteracting and, due to hydrodynamic interactions and

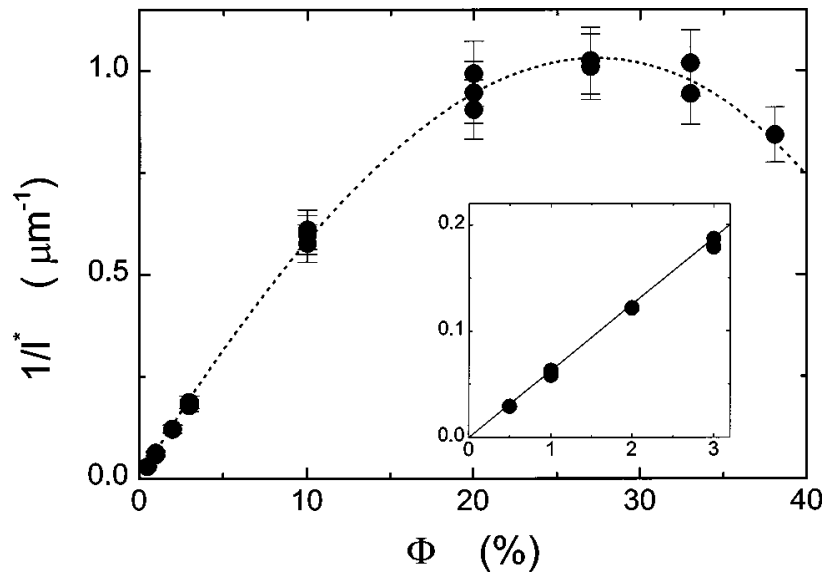

FIG. 6. Dependence of the inverse transport mean free path on the volume fraction $\Phi$ for $208 \mathrm{~nm}$ diameter barium titanate particles. The inset displays the linear increase of $1 / l^{*}$ for small volume fractions where $1 / l^{*}$ was determined by $C_{1}(t)$ measurements [Eq. (11)], whereas for higher volume fractions the values are based on measurements of the totally transmitted light intensity. The dotted line is a guide to the eye for the experimentally determined values of $1 / l^{*}$.

positional correlations, $\tau_{0}$ becomes dependent on the volume fraction. ${ }^{21,22}$ Thus, we decided to determine $l^{*}$ independently by measuring the total transmitted light intensity $T_{0}$ relative to samples of sufficiently low concentration $[\Phi \leqslant 2 \%$, where $\left.\tau_{0} \simeq \tau_{0}(0)\right] .{ }^{17,21}$ In order to suppress the influence of surface reflectivity, the measurements were carried out with the samples suspended in a tank filled with water (index matching bath). For sufficiently thick samples $\left(L>6-8 l^{*}\right)$ the transmission coefficient is then given by ${ }^{23}$

$$
T_{0}=\frac{5}{3}\left(\frac{l^{*}}{L}\right)\left[1+\frac{4}{3}\left(\frac{l^{*}}{L}\right)\right]^{-1}
$$

Figure 6 shows the concentration dependence of the inverse transport mean free path $1 / l^{*}$. After a linear increase for small volume fractions (inset Fig. 6) we observe a flattening of the slope followed by a maximum at $\Phi \approx 27 \%$, corresponding to a minimum value of $l^{*}=0.98 \pm 0.08 \mu \mathrm{m}$, whereas for higher values of $\Phi, 1 / l^{*}$ decreases again. This behavior is in qualitative agreement with the behavior predicted by Mie theory for monodisperse hard spheres of this size. Due to the influence of positional correlations $1 / l^{*}$ is reduced from its value expected for a noncorrelated system. $^{21,22}$ With the knowledge of $l^{*}$ we could determine $\tau_{0}(\Phi)$ from measurements of $C_{1}(t)$ [Eq. (11)] at the same samples. A decay of $1 / \tau_{0}(\Phi)$ with increasing volume fraction $\Phi$ is observed (data not shown) and we find a value of $\tau_{0}(27 \%)=2.5 \pm 0.3 \mathrm{~ms}$.

Before each experiment the thickness $L$ of the sample cell was measured by comparing the intensity-intensity correlation function $C_{1}(t)$ of the sample to the correlation function measured on a slab of known thickness [using a commercially available glass cell of thickness $(100 \pm 5) \mu \mathrm{m}]$. Because $l^{*}$ and $\tau_{0}$ are identical in both measurements, the accuracy in $L$ does not depend on the actual values of $l^{*}$ and $\tau_{0}$, but only on the error bar of the reference cell thickness [Eq. (11)]. 
Laterally, the samples turned out to be homogeneous, $L$ varying typically less than $2-5 \%$ within $200 \mu \mathrm{m}$ around the chosen sample spot. To check the stability of the sample, we repeated the measurement of $C_{1}(t)$ each time the samplelens distance, i.e., the beam spot size $w$, was changed. Due to sedimentation, drying, and remaining aggregation in this confined geometry we observed that $C_{1}(t)$ decays somewhat slower after a certain time. We stopped the run, if for fixed $\tau_{0}$ the ratio $L / l^{*}$ in Eq. (11) deviated by more than $5 \%$ from its initial value, which was typically the case after $2-3 \mathrm{~h}$. Due to this time limit, we chose a single run time of 10-30 min for one beam spot size $w$, depending on the signal strength, i.e., the conductance $g$, and the desired accuracy.

\section{Internal reflections}

When measuring the integrated transmission of a multiple-scattering layer, as for the determination of $C_{2}(t)$, most of the light entering the sample will not be transmitted but reflected, i.e., the transmission coefficient of a slab is generally small, i.e., $T_{0}<0.2$ [Eq. (15)], in the multiplescattering regime $\left(L / l^{*}>8\right)$. Part of this backscattered light is reflected again at the outer surface of the sample cell by angular-dependent reflection. If the sample cell is surrounded by air, due to the high index mismatch glass/air, $\Delta n \approx 1.5$, almost all of the reflected light will be due to total reflection. The reflection coefficient at the outer surface $R_{s}$ due to total reflection can be calculated. Assuming the photon cloud inside the sample to be an ideal diffuser in the backward direction, the light emission is uniform and the light current in a direction $\vartheta$ is given by $J=J_{0} \cos (\vartheta)$. Integrating the light flux over the angles of transmission, $\vartheta<\vartheta_{\text {crit }}$, and total reflection, $\vartheta>\vartheta_{\text {crit }}$, yields

$$
R_{s}=1-\sin ^{2}\left(\vartheta_{\text {crit }}\right)
$$

Hence, for a glass/air interface with an angle of total reflection of $\vartheta_{\text {crit }} \simeq 42^{\circ}$ we find a reflection coefficient of $R_{s} \simeq 0.55$. Reentering the sample this light is much less spatially confined and gives only a negligible contribution to the $C_{2}$ correlations. This background noise contribution, $T_{\text {background }}$, due to total reflection at the outer surface can be calculated easily by summing up all reflection contributions:

$$
\begin{aligned}
T_{\text {background }} & =\sum_{n=1}^{\infty}\left[\left(1-T_{0}\right) R_{s}\right]^{n} T_{0} \\
& =\left[\frac{1}{1-\left(1-T_{0}\right) R_{s}}-1\right] T_{0} .
\end{aligned}
$$

Consequently this background noise gives a significant uncorrelated contribution to the measured integrated transmission and, therefore, increases the measured values of the conductance $g$. Already for relatively thin samples of $L=8 l^{*} \quad\left(T_{0}=0.17\right)$, the background noise $T_{\text {background }}$ $=0.82 T_{0}$ is of the same order as $T_{0}$, increasing rapidly to its maximum of $T_{\text {background }}=1.2 T_{0}$ for thick samples. ${ }^{24}$

In order to totally suppress this background light intensity we replaced the incoming transparent glass window of the sample cell with a highly absorbing glass plate (Schott,

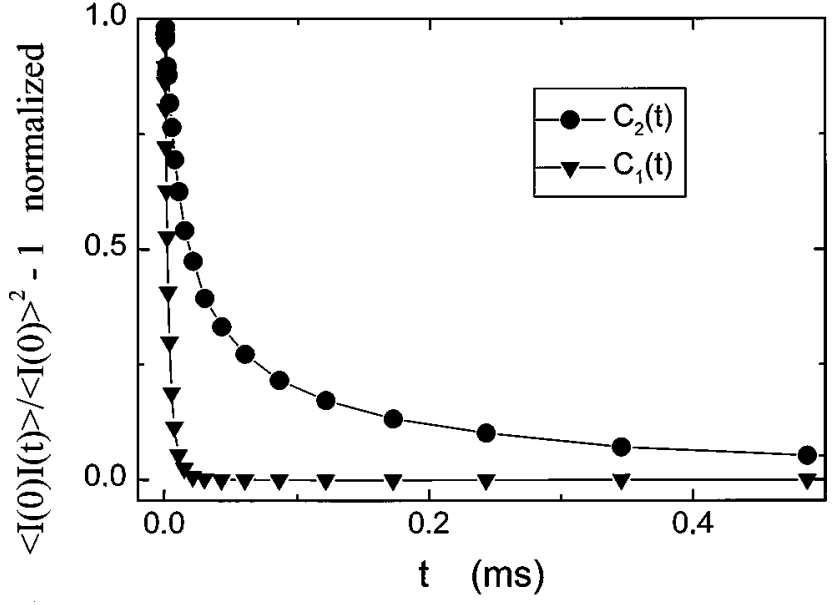

FIG. 7. Comparison of the functional dependence of the $C_{1}(t)$ and the $C_{2}(t)$ correlation functions for the transmission of light through a slab of thickness $L=19.6 \mu \mathrm{m}\left[l^{*}=0.98 \mu \mathrm{m}, w=11.6\right.$ $\mu \mathrm{m}]$.

$T=0.005$ ), normally used as a neutral filter. By increasing the input laser power we compensated the absorption loss to obtain the same incident light intensity at the sample surface. The reflected light is now absorbed within the absorbing glass plate, hence eliminating the background light intensity. Experimentally we observed an increase in $1 / g$ of approximately a factor of 2 , as compared to samples with a simple glass window in agreement with the above considerations.

\section{RESULTS AND DISCUSSION}

\section{A. Observation of long-range $C_{2}$ correlations in temporal intensity fluctuations}

Using the setup described above we were able to measure long-range correlations of temporal fluctuations with high accuracy. Measurements were carried out for different thicknesses $L$, beam spot sizes $w$, and values of the transport mean free paths $l^{*}$.

First we discuss some general features of these long-range correlations. Figure 5 shows the intensity-intensity correlation functions for three different beam spot sizes, with $l^{*}=0.98 \mu \mathrm{m}$. The maximum signal observed for this film thickness $L=36.7 \mu \mathrm{m}$ is of the order of $\langle I(0) I(0)\rangle /\langle I(0)\rangle^{2}$ $-1 \approx 2 \times 10^{-4}$ corresponding to a conductance of $g \approx 5000$. Nevertheless, we are able to clearly separate the long-range $C_{2}(t)$ correlations from the intrinsic noise as shown in Fig. 5. With increasing beam spot size the magnitude of $C_{2}(t)$ decays sharply and disappears for large values of $w$.

A comparison of the intensity-intensity correlation function $C_{1}(t)$ detected on a single speckle spot (one outgoing light mode) with the integrated transmission $C_{2}(t)$ (all outgoing modes) is displayed in Fig. 7. Clearly the latter function decays much slower and over a much broader time scale than the first one.

\section{B. Contribution from the boundary layer}

We consider now in more detail the dependence of $1 / g$ on $l^{*}$ and explore the limits of $w \approx l^{*}$, where an increased in- 


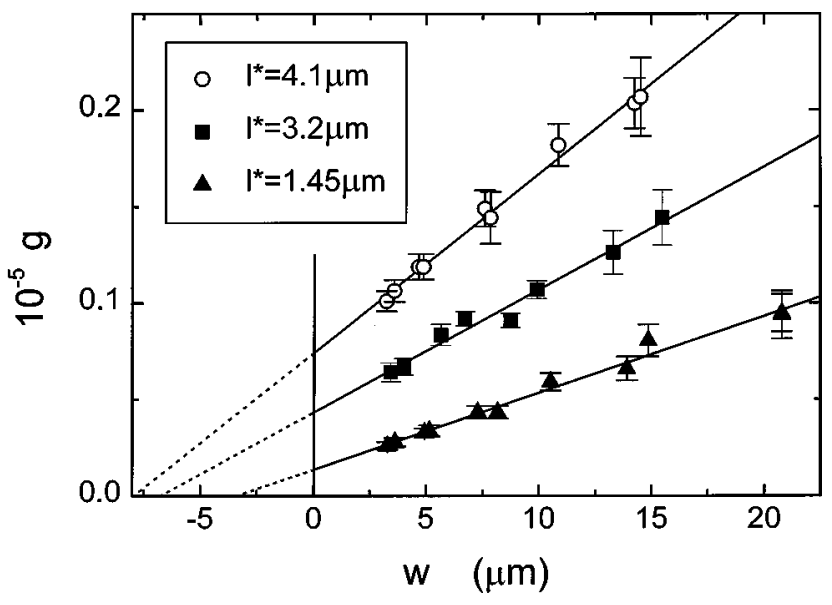

FIG. 8. Measurements of the conductance $g$ vs the beam spot size $w$ show that $g$ values extrapolated for $w \rightarrow 0, g_{\text {min }}$ are nonzero and a distinct function of the transport mean free path $l^{*}$. A minimum beam spot size, $w_{\text {min }}$, can be defined by extrapolation of $g(w) \rightarrow 0$ (dotted lines). The thickness of the films $(L=90 \pm 10 \mu \mathrm{m})$ is in all cases much larger than the beam spot size $w$.

fluence of the light propagating in a layer near the sample surface is expected. In the case of $C_{2}$ correlations, crossing of light paths near the incoming surface results in short "active" scattering paths, after which no further dephasing occurs. These short active paths are responsible for the longtime tail of the correlation function $C_{2}(t)$.

A significant influence of this boundary layer on $g$ as probed by $C_{2}(t)$ is expected when the beam size $w$ is of the order of the transport mean free path $l^{*}$, which is the length scale over which the incident light is randomized. Figure 8 shows the dependence of $g$ on $w$ for thick films ( $L=90 \pm 10 \mu \mathrm{m})$ of colloidal suspensions of different $l^{*}$. The concentration $\Phi$ of the suspensions is in all cases $11 \%$ or lower, therefore $n \simeq n_{\text {water }} \simeq$ const. In this range $(w / L<0.2)$ $g / L$ is in good approximation linear in $(w / L)$ as can be shown by expanding Eq. (5) in the limit $w / L \rightarrow 0$ :

$$
g \simeq \frac{8 \alpha}{5} w
$$

The experimental results (Fig. 8) clearly show that the conductance $g$ does not tend to zero for small values of $w$, but reaches a well defined minimum value $g_{\min }\left(l^{*}\right)$. Apparently, the light incident on the slab does not contribute to the long-range correlations before it is scattered at least once inside a surface layer, hence broadening the beam spot. Although an accurate theoretical description is not available yet, we can discuss the problem on a qualitative basis. The only length scale entering the problem is the transport mean free path $l^{*}$, suggesting that the thickness of the boundary layer is proportional to $l^{*}$, and therefore $w_{\min } \sim l^{*}$ as well. With Eq. (19) and Eq. (6) this yields

$$
g_{\min } \simeq \frac{8 \alpha}{5} w_{\min } \propto \alpha l^{*} \propto n^{2} l^{* 2} .
$$

Since $n \simeq$ const we expect from this argument that $g_{\text {min }}$ should vary proportional to $l^{* 2}$. Experimentally the agreement with Eq. (20) is good (Fig. 9). The minimum effective

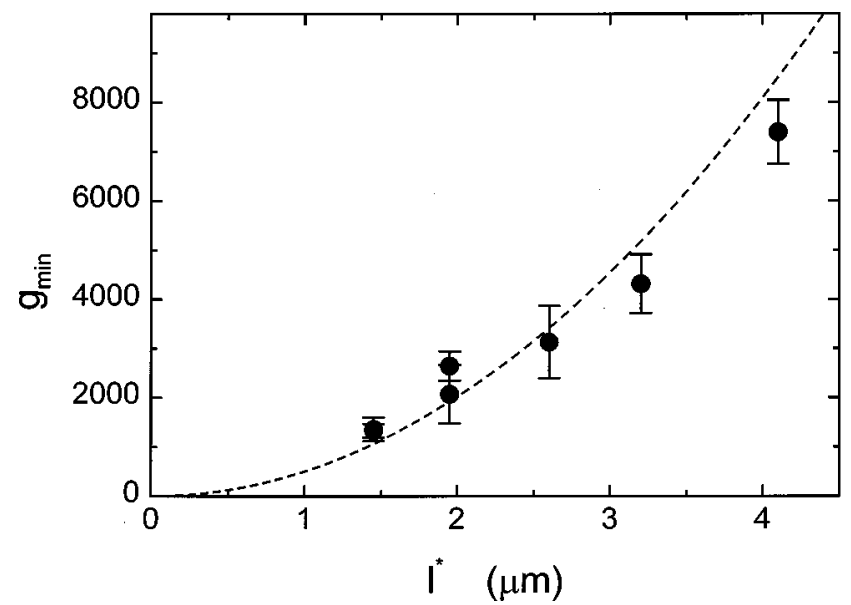

FIG. 9. Minimum value $g_{\text {min }}$ of the conductance determined by extrapolation of $g(w)$ to $w \rightarrow 0$ according to Eq. (19) (see Fig. 8). $g_{\text {min }}$ increases roughly quadratically with the transport mean free path $l^{*}$ (dashed line).

beam size, calculated from Eq. (20), is determined to $w_{\text {min }}=(2.4 \pm 0.5) l^{*}$, which is of the expected order. This result suggests that in fact the photon intensity distribution is broadened by scattering in a surface layer of thickness $(1-2) l^{*}$.

\section{Scaling behavior of the conductance $g$}

According to Eq. (5) the conductance $g$ divided by $L$ should depend solely on the ratio of the beam spot size and sample thickness $w / L$, independently of the actual values of $w$ and $L$. For this reason, the measured values of the conductance $g / L$ are predicted to follow a master curve. For values of $w \ll L$ the rescaled conductance $g / L$ increases linearly with $w / L$, whereas for large ratios $w / L \gg 1$ the quadratic law [Eq. (3)] should be recovered: $g / L \sim(w / L)^{2}$.

We take into account the broadening inside the surface layer, as discussed above, by replacing the beam size $w$ by an effective beam size:

$$
w_{\mathrm{eff}}=\left(w_{\min }+w\right)=2.4 l^{*}+w
$$

The conductance $g$ for films of different thicknesses $(L=8,19.6,36.7,40.2 \mu \mathrm{m})$ and effective beam spot sizes from 14 to about $60 \mu \mathrm{m}$ was determined for a single minimum transport mean free path of $l^{*}=0.98 \mu \mathrm{m}$. Over the explored range of $w_{\text {eff }} / L$, the measured ratios $g / L$ are in good agreement with the scaling prediction Eq. (5) (Fig. 10) with only one adjustable parameter $\alpha=149 \pm 15[1 / \mu \mathrm{m}]$.

Another feature of Eq. (5) [see also Eq. (9)] is illustrated in Fig. 11. For $L / w_{\text {eff }} \gg 1$ the magnitude of the $C_{2}$ correlations, i.e., the inverse conductance $1 / g$, should become independent of $L$. This is due to the broadening of the beam inside the sample (Fig. 2). If $L$ is much larger than $w_{\text {eff }}$, the width of the photon cloud deep inside the sample becomes so large, that the crossing probability is very small in most of the sample except the region of thickness of order $\approx w_{\text {eff }}$ near to the entrance surface. Increasing the thickness yields only asymptotically small increases in $1 / g$ (Fig. 11).

We now compare quantitatively the value of $\alpha$ obtained from our measurements to the theoretical prediction [Eq. 


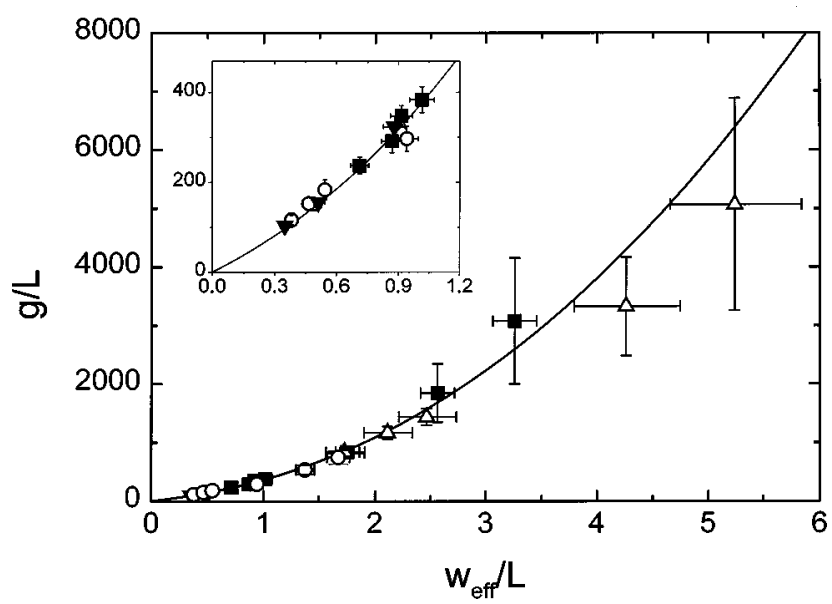

FIG. 10. Scaling dependence of the reduced conductance $g / L$ on the reduced beam spot size $w_{\text {eff }} / L$. The measured values for four different film thicknesses $L=8,19.6,36.7,40.2 \mu \mathrm{m}$ follow a master curve [Eq. (5), solid line] with $\alpha=149 \pm 15$ the only adjustable parameter.

(5)], and to the values obtained in the case of frequency correlations. $^{12}$ This prefactor represents the geometryindependent part of the expression for $g$, i.e., its dependence on $l^{*}$ and the wavelength $\lambda / n$, which finally determines the number of light modes inside the sample.

The wave vector $k_{0}$ is determined simply by the wavelength of light in the medium $k_{0}=2 \pi(n / \lambda)$. While the wavelength $\lambda=457.9 \mathrm{~nm}$ of the incoming light is given, there is some uncertainty concerning the refractive index $n$. The phase velocity inside the medium is reduced due to the presence of the scatterers, making the effective refractive index higher, as compared to the surrounding medium. Brewster angle measurements at similar systems $(260 \mathrm{~nm}$ $\mathrm{TiO}_{2}$ in air) by den Outer ${ }^{25}$ indicate that the effective refractive index is located between the refractive index of the surrounding medium and the geometrical average:

$$
n \approx\left[\Phi * n_{\text {particle }}+(1-\Phi) n_{\text {medium }}+n_{\text {medium }}\right] / 2 .
$$

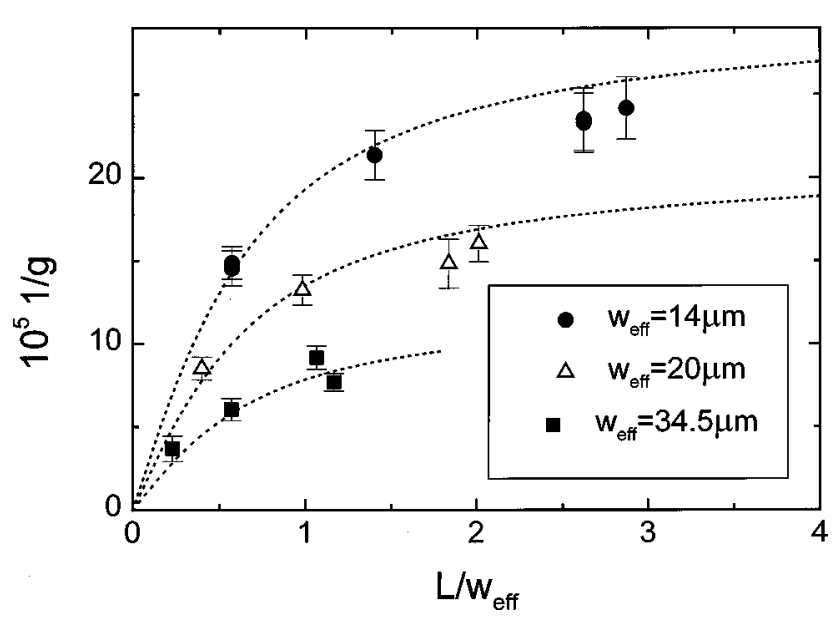

FIG. 11. Dependence of the inverse conductance $1 / g$ on the slab thickness $L$. The values for three different beam spot sizes $w_{\text {eff }}=14$, 20, $34.5 \mu \mathrm{m}$ are plotted with the corresponding theoretical lines (dotted), Eq. (5), using $\alpha=149$.

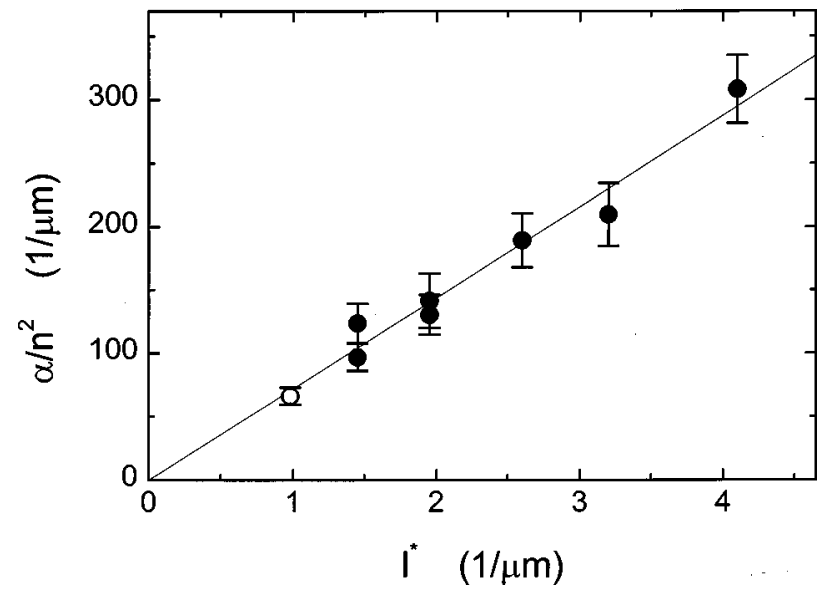

FIG. 12. The values of $\alpha / n^{2}$ show the expected linear dependence on $l^{*}$. The $\alpha$ values are determined from the slope of measured $g(w)$ curves (Fig. 8, full circles) and from the analysis of the scaling behavior (Fig. 10, open circle) for the smallest $l^{*}=0.98 \mu \mathrm{m}$ and the refractive index $n$ was estimated using Eq. (22).

In our case $n \approx 1.5 \pm 0.15$ since $n_{\text {medium }}=n_{\text {water }}=1.33$. It is difficult to measure this quantity with high precision and no theory is available, which is the reason for the large error bar. Substituting this value into Eq. (6) yields $\alpha \approx 138 \pm 27[1 /$ $\mu \mathrm{m}]$. The experimentally determined value of $\alpha=149 \pm 15[1 /$ $\mu \mathrm{m}]$ is found to be in excellent agreement. This result confirms the conclusions of de Boer et al. ${ }^{12}$ drawn from frequency correlation measurements at a system of $d \approx 220 \mathrm{~nm}$ $\mathrm{TiO}_{2}$. Their system parameters are $\lambda=600 \mathrm{~nm}, l^{*}=0.7 \mu \mathrm{m}$, $n \approx 1.3 \pm 0.18$ which yields $\alpha=43 \pm 10$, compared to an experimental value of $\alpha=45 \pm 5$.

Finally, we were able to confirm the predicted linear dependence of $g$ on the transport mean free path $l^{*}$ [Eq. (6)]. Figure 12 shows the values of $\alpha / n^{2}$ determined from the slope of the $g(w)$ curves (Fig. 8). In addition is shown the value determined for $l^{*}=0.98 \mu \mathrm{m}$ as described above. A linear fit yields $\alpha / n^{2}=(71 \pm 9) * l^{*}$, compared to $\alpha / n^{2}$ $=63 * 1 *$ from theory [Eq. (6)].

\section{Time-dependent shape of the correlation function $C(t)$}

Finally we discuss the time dependence of the correlation function $C_{2}(t)$. Crossing of light paths can occur at any point inside the sample, its probability being determined only by the effective lateral extension of the photon cloud as discussed above. Consequently the length distribution of light paths which build up $C_{2}(t)$ is much broader than that controlling the short-range $C_{1}(t)$ correlations. $C_{2}(t)$ is therefore expected to show a much slower and broader decay than $C_{1}(t)$. A semilogarithmic plot, Fig. 13, clearly reveals the different characteristics of the $C_{1}(t)$ and the $C_{2}(t)$ correlation functions as observed in our experiments. Whereas $C_{1}(t)$ shows a sharp, nearly exponential decay, $C_{2}(t)$ decays over nearly three decades in time.

The dependence of the crossing probability on the position of the crossing event inside the sample [i.e., the depth $z$, discussed above (Fig. 2)] also affects the time dependence of $C_{2}(t)$. Entering the sample with a beam size $w$, the lateral intensity distribution spreads out on its way through the 


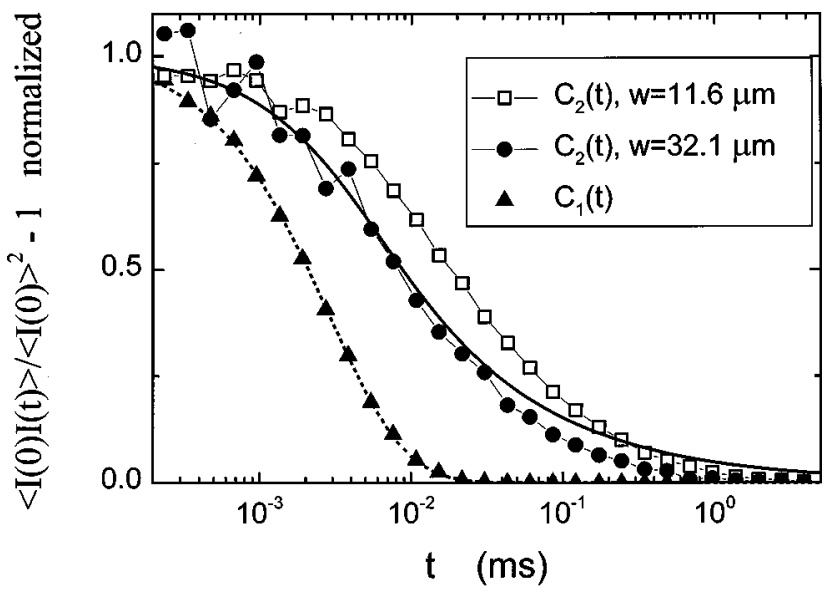

FIG. 13. Intensity-intensity correlation function $C_{1}(t)$ for single speckle detection (triangle up). Perfect agreement with Eq. (11) is found (dotted line), with $L=19.6 \mu \mathrm{m}$ the only adjustable parameter. The $C_{2}(t)$ decay is shown to depend on the beam spot size $w$. The smaller $w$ the slower the decay. The solid line is the dependence predicted in the limit $w \gg L$ [Eq. (13)] with no adjustable parameter.

sample. Henceforth the probability of crossing paths decays, as can be seen from Eq. (3), since $g$ is proportional to the square of the lateral dimension of the sample. For $w<L$ most correlations are created close to the incoming surface, where the lateral dimension of the illuminated sample is comparable to $w$. For depths larger than $z \approx w$ (compare also to Fig. 11 ), the width of the beam has already increased considerably and from this point on the contributions to $C_{2}(t)$ are small. Short light paths dominate $C_{2}(t)$ and the decay is much slower than expected for a homogeneously illuminated sample.

If the beam spot size is larger than the sample thickness, $w>L$, the lateral dimensions of the intensity distribution are only slightly changed inside the sample and light paths from all depths contribute to $C_{2}(t)$. For this latter case the theory of Berkovits and Feng ${ }^{10}$ should provide a good approximation. The measured correlation functions show both these features. It can be clearly seen from Fig. 13 that for a sample of thickness $L=19.6 \mu \mathrm{m}$ a small beam spot size $w=11.6 \mu \mathrm{m}$ results in a much slower decay as compared to the large beam spot size $w=32.1 \mu \mathrm{m}$. For smaller ratios of $w / L$ we find this difference to be even more pronounced. By increasing the beam size the correlation function decays faster. In this case the experimentally determined $C_{2}(t)$ cor- relation function is in good agreement with the prediction of Berkovits and Feng over a wide range of correlation times. For long correlation times the theory and experiment deviate; while theory predicts an algebraic $t^{-1 / 2}$ tail of the correlation function, experimentally we observe a much faster decay. We attribute this downward deviation to surface effects not accurately treated in current theories as discussed above.

\section{SUMMARY AND CONCLUSIONS}

By studying the light intensity fluctuations of the integrated light transmission through a slab we were able to provide evidence for the existence of long-range temporal correlations. Based on a series of measurements with different sample thicknesses $L$, beam spot sizes $w$, and transport mean free paths $l^{*}$, we were able to confirm the scaling predictions for the optical conductance $g$. The time dependence of the long-ranged $C_{2}(t)$ correlation function shows good overall agreement with theory, but we observe a distinct downward deviation of the data at long times, i.e., $t>\tau_{0} / 20$. This finding indicates a cutoff of the contribution of short scattering paths to $C_{2}(t)$. The study of the conductance $g$ for extremely small values of $w \approx l^{*}$ delivers further evidence for this assumption. It is argued that at least one scattering event is required before the event of crossing of two paths and, therefore, the correlated sections of paths are cut off at the low path length side at 1 to $2 l^{*}$. At the effective depth where correlated light paths start to contribute to $C_{2}(t)$ the width of the beam has already increased due to the scattering in the boundary layer, which leads to a minimum conductance $g_{\min }\left(l^{*}\right)$ even for $w \rightarrow 0$. We conclude that improved theoretical models are necessary to describe accurately the coupling of light into the random medium and the spatial distribution of path crossing events near the surface. Measurements of $C_{2}(t)$ may provide sensitive probes to test the accuracy of such models.

\section{ACKNOWLEDGMENTS}

We would like to thank Marcel Boehmer and Gero Decher for useful comments concerning the stabilization of the barium titanate suspensions. F.S. acknowledges financial support by the Deutscher Akademischer Austauschdienst (DAAD) through the HSPII/AUFE program. W.H. acknowledges financial support by the European Community in the frame of the Human Capital and Mobility program, Contract No. ERBCHRXCT930373.
${ }^{1}$ S. John, Phys. Today 44 (5), 32 (1991).

${ }^{2}$ M. P. van Albada and A. Lagendjik, Phys. Rev. Lett. 55, 2692 (1985).

${ }^{3}$ P. E. Wolf and G. Maret, Phys. Rev. Lett. 55, 2696 (1985).

${ }^{4}$ Scattering and Localization of Classical Waves in Random Media, edited by P. Sheng (World Scientific, Singapore, 1990).

${ }^{5}$ Photonic Band Gaps and Localization, Vol. 308 of Nato Advanced Study Institute, Series B: Physics, edited by C. M. Soukoulis (Plenum, New York, 1993).
${ }^{6}$ Ping Sheng, Introduction to Wave Scattering, Localization, and Mesoscopic Phenomena (Academic, Boston, 1995).

${ }^{7}$ G. Maret, in Mesoscopic Quantum Physics, edited by E. Akkermans, G. Montambaux, J-L. Pichard, and J. Zinn-Justin (Elsevier Science B.V., North-Holland, Amsterdam, 1995), p. 147.

${ }^{8}$ S. Feng, C. Kane, P. A. Lee, and A. D. Stone, Phys. Rev. Lett. 61, 834 (1988).

${ }^{9}$ S. Feng and P. A. Lee, Science 251, 633 (1991). 
${ }^{10}$ R. Berkovits and S. Feng, Phys. Rep. 238, 135 (1994).

${ }^{11}$ M. P. van Albada, J. F. de Boer, and A. Lagendijk, Phys. Rev. Lett. 64, 2787 (1990).

${ }^{12}$ J. F. de Boer, M. P. van Albada, and A. Lagendijk, Phys. Rev. B 45, 658 (1992).

${ }^{13}$ A. Z. Genack, N. Garcia, and W. Polkosnik, Phys. Rev. Lett. 65, 2129 (1990).

${ }^{14}$ R. Pnini and B. Shapiro, Phys. Rev. B 39, 6986 (1989).

${ }^{15}$ The assumption of $w(z) \approx w+\beta z$ is jusitified as long as the influence of the surface at the transmission side can be neglected, i.e., for $z \leqslant L / 2$.

${ }^{16}$ D. A. Weitz and D. J. Pine, in Dynamic Light Scattering, edited by W. Brown (Oxford, New York, 1993), Chap. 16, pp. 652720.

${ }^{17}$ P. D. Kaplan, M. H. Kao, A. G. Yodh, and D. J. Pine, Appl. Opt. 32, 3828 (1993).

${ }^{18}$ K. Schaetzel, R. Kalstroem, B. Stampa, and J. Ahrens, J. Opt.
Soc. Am. B 6, 937 (1989).

${ }^{19}$ Y.-S. Her, E. Matijević, and M. C. Chon, J. Mater. Res. 10, 3106 (1995).

${ }^{20}$ A. W. M. de Laat, Ph.D. thesis, University of Wageningen, The Netherlands, 1995.

${ }^{21}$ S. Fraden and G. Maret, Phys. Rev. Lett. 65, 512 (1990).

${ }^{22}$ X. Qiu, X. L. Wu, J. Z. Xue, D. J. Pine, D. A. Weitz, and P. M. Chaikin, Phys. Rev. Lett. 65, 516 (1990).

${ }^{23}$ A. Ishimaru, Wave propagation and Scattering in Random Media (Academic, New York, 1978), Vol 1.

${ }^{24}$ Due to the rapid increase of $T_{\text {background }}$ to its maximum value, surface reflections are of much less importance, as compared to the measurements of $g$, if only the ratio of $T_{0}$ between samples of different $L / l^{*}$ is determined, e.g., for measurements of $l^{*}$ (Refs. 21 and 22).

${ }^{25}$ Peter den Outer, Ph.D. thesis, University of Amsterdam, The Netherlands, 1995 